\title{
Interrelationships between Cardiac Performance Measurements
}

\author{
Glarence M. Agress, M.D. and Stanlcy Wegner
}

\begin{abstract}
A comparison of cardiovascular performance measurements in current use was made in anesthetized dogs at rest and after administration of isoproterenol. The flow-related variables-stroke work, stroke power, stroke volume, cardiac output and mean ejection rate showed the best correlations, while the time tension index, ventricular pressure derivative, heart rate and mean systolic pressure showed no consistent correlations either with the stroke index or with each other. It was concluded that the effects of altered hemodynamics must be considered before using different cardiac performance measurements interchangeably.
\end{abstract}

THE most widely accepted methods for assessing the performance of the 1 heart have been to cxaminc its volume output, its work performed or power generated. These measurements have been used as standards for gauging cardiovascular stress. ${ }^{1,2)}$ More recently, additional indices of cardiac performance have been described which include the tension time index, ${ }^{3)}$ mean ejection rate ${ }^{4}$ and pressure derivative. ${ }^{5)}$ These have been considered to change in parallel with the standard cardiac performance measurements. ${ }^{61,7)}$ This experimental study was undertaken to examine the interrelationships between measurements of cardiac performance at rest and after alteration of hemodynamics.

\section{Methods}

Ten mongrel dogs ranging in weight from 15 to $24 \mathrm{Kg}$, anesthetized with intravenous pentobarbital, $60 \mathrm{mg}$. $/ \mathrm{Kg}$. were used in the study. Mercury manometer calibrated catheters were passed to the aortic root and into the left ventricle via the right and left carotid arteries for the recording of aortic and left ventricular pressures. Cardiac output was determined by the dye dilution method using cardiogreen dye and an automatic computing device. ${ }^{*}$ The first derivation of intraventricular pressure was obtained using a Dymec operational amplifier. ${ }^{*}$ These parameters were recorded on an Electronics for Medicine photographic recorder*** using

\footnotetext{
From the Institute for Medical Research, Gedars of Lebanon Hospital, Los Angeles, California. This study was supported by Grant $\mathrm{N}_{\mathrm{S}} \mathrm{G}-289 / 05-12-001$ : from National Aeronautics and Space Administration.

Received for publication October 12, 1965.

* Gilford Model 104

** Dymec Model 2460A

*** Electronics for Medicine Model DR-8
} 
a paper speed of $200 \mathrm{~mm}$. $/ \mathrm{sec}$. From these recordings, calculations were made as follows :

\author{
Measurement \\ Mean Systolic Pressure (MSP)
}

Tension Time Index (TTI)

Maximum Ventricular Pressure Derivative $(\mathrm{dP} / \mathrm{dt})$

Stroke Volume Index (SVI)

Stroke Work Index (SWI)

Stroke Power Index (SPI)

Mean Ejection Rate (MER)

\author{
Calculation \\ Area under Aortic Curve \\ Duration of Ejection \\ + Diastolic Press. \\ Area under Aortic Curve \\ By Differentiation** \\ $\frac{\text { Cardiac Index }}{\text { Heart Rate }}$ \\ SVI times MSP \\ $\frac{\text { SWI }}{\text { Ejection Time }}$ \\ Stroke Volume \\ Ejection Time
}

Units

$\mathrm{mm} . \mathrm{Hg}$

mm. $\mathrm{Hg}$-secs.

$\mathrm{mm} . \mathrm{Hg} / \mathrm{sec}$.

ml. $/ \mathrm{Kg}$.

mm.Hg-ml./Kg.

mm.Hg-ml./Kg. secs.

$\mathrm{ml} . / \mathrm{sec}$.

Paired samples of the above measurements were made in resting states and with infusions of isoproterenol (Isuprel), given in a drip of dextrose and water $(0.4 \mathrm{mg}$./L.) at a rate of $60 \mathrm{drops} / \mathrm{min}$. for 5 to $10 \mathrm{~min}$. This drug was chosen because it has been reported to simulate the effects of exercise. ${ }^{8)}$ The data were analyzed by performing linear regression analyses of each of these per- formance measurements on the stroke volume indices, ${ }^{91}$ Independent analyses were performed for resting measurements and after isoproterenol exhibition.

\section{Results}

The data obtained from the performance measurements are summarized in Table $\mathrm{I}$. The ranges of the variables at rest were:

MSP : $\quad 83-136 \mathrm{~mm} . \mathrm{Hg}$

TTI : $\quad 17.9-36.5 \mathrm{~mm} . \mathrm{Hg}$ sec.

SVI : $\quad 0.63-2.07 \mathrm{ml} . / \mathrm{Kg}$.

SWI : $\quad 49-215 \mathrm{~mm} . \mathrm{Hg}-\mathrm{ml} . / \mathrm{Kg}$.

SPI : $\quad 393-1,319 \mathrm{~mm} . \mathrm{Hg}-\mathrm{ml} . / \mathrm{Kg}$. sec.

$\mathrm{dP} / \mathrm{dt}: \quad 1,000-2,700 \mathrm{~mm} . \mathrm{Hg} / \mathrm{sec}$.

HR : $\quad 96-170$ beats/min.

$\mathrm{CO}: \quad 1.83-3.90 \mathrm{~L} . / \mathrm{min}$.

MER : $76-190 \mathrm{ml} . / \mathrm{sec}$.

After infusion of isoproterenol the ranges were:

MSP : $\quad 72-119 \mathrm{~mm} . \mathrm{Hg}$

TTI : $\quad 17.3-43.8 \mathrm{~mm} . \mathrm{Hg}$-scc.

SVI : $\quad 0.67-2.27 \mathrm{ml} . / \mathrm{Kg}$. 
Table I

\begin{tabular}{|c|c|c|c|c|c|c|c|c|c|c|}
\hline & & MSP & TTI & SVI & SWI & SPI & $\mathrm{dP} / \mathrm{dt}$ & HR & $\mathrm{CO}$ & MER \\
\hline \multirow[t]{2}{*}{1.} & $\mathrm{C}$ & $\begin{array}{l}104 \\
101\end{array}$ & $\begin{array}{l}20.4 \\
25.0\end{array}$ & $\begin{array}{l}2.07 \\
1.97\end{array}$ & $\begin{array}{l}215 \\
199\end{array}$ & $\begin{array}{l}1,319 \\
1,191\end{array}$ & $\begin{array}{l}2,700 \\
2,560\end{array}$ & $\begin{array}{l}125 \\
125\end{array}$ & $\begin{array}{l}3.9 \\
3.7\end{array}$ & $\begin{array}{l}190 \\
177\end{array}$ \\
\hline & I & $\begin{array}{l}82 \\
72\end{array}$ & $\begin{array}{l}32.1 \\
40.8\end{array}$ & $\begin{array}{l}2.27 \\
2.20\end{array}$ & $\begin{array}{l}186 \\
158\end{array}$ & $\begin{array}{r}1,148 \\
863\end{array}$ & $\begin{array}{l}2,430 \\
2,560\end{array}$ & $\begin{array}{l}115 \\
123\end{array}$ & $\begin{array}{l}4.0 \\
4.1\end{array}$ & $\begin{array}{l}214 \\
182\end{array}$ \\
\hline \multirow[t]{2}{*}{2.} & $\mathrm{C}$ & $\begin{array}{l}96 \\
99\end{array}$ & $\begin{array}{l}19.1 \\
19.6\end{array}$ & $\begin{array}{l}0.63 \\
0.60\end{array}$ & $\begin{array}{l}60 \\
59\end{array}$ & $\begin{array}{l}408 \\
393\end{array}$ & $\begin{array}{l}1,450 \\
1,450\end{array}$ & $\begin{array}{l}170 \\
159\end{array}$ & $\begin{array}{l}2.02 \\
1.81\end{array}$ & $\begin{array}{l}81 \\
76\end{array}$ \\
\hline & 1 & $\begin{array}{l}101 \\
106\end{array}$ & $\begin{array}{l}20.9 \\
22.3\end{array}$ & $\begin{array}{l}0.67 \\
0.78\end{array}$ & $\begin{array}{l}68 \\
83\end{array}$ & $\begin{array}{l}503 \\
653\end{array}$ & $\begin{array}{l}1,740 \\
1,670\end{array}$ & $\begin{array}{l}175 \\
150\end{array}$ & $\begin{array}{l}2.25 \\
2.23\end{array}$ & $\begin{array}{r}95 \\
117\end{array}$ \\
\hline \multirow[t]{2}{*}{3.} & $\mathrm{C}$ & $\begin{array}{l}117 \\
125\end{array}$ & $\begin{array}{l}17.9 \\
19.3\end{array}$ & $\begin{array}{l}0.99 \\
0.99\end{array}$ & $\begin{array}{l}116 \\
125\end{array}$ & $\begin{array}{r}928 \\
1,000\end{array}$ & $\begin{array}{l}2,000 \\
2,170\end{array}$ & $\begin{array}{l}140 \\
140\end{array}$ & $\begin{array}{l}2.39 \\
2.40\end{array}$ & $\begin{array}{l}137 \\
137\end{array}$ \\
\hline & I & $\begin{array}{l}101 \\
115\end{array}$ & $\begin{array}{l}21.6 \\
22.1\end{array}$ & $\begin{array}{l}1.16 \\
0.70\end{array}$ & $\begin{array}{r}117 \\
80\end{array}$ & $\begin{array}{l}936 \\
707\end{array}$ & $\begin{array}{l}2,350 \\
3,130\end{array}$ & $\begin{array}{l}168 \\
177\end{array}$ & $\begin{array}{l}3.32 \\
2.14\end{array}$ & $\begin{array}{l}158 \\
107\end{array}$ \\
\hline \multirow[t]{2}{*}{4.} & $\mathrm{C}$ & $\begin{array}{l}115 \\
136\end{array}$ & $\begin{array}{l}29.2 \\
33.5\end{array}$ & $\begin{array}{l}1.52 \\
1.52\end{array}$ & $\begin{array}{l}175 \\
207\end{array}$ & $\begin{array}{r}994 \\
1,182\end{array}$ & $\begin{array}{l}2,100 \\
2,300\end{array}$ & $\begin{array}{l}110 \\
113\end{array}$ & $\begin{array}{l}2.57 \\
2.66\end{array}$ & $\begin{array}{l}133 \\
134\end{array}$ \\
\hline & I & $\begin{array}{l}106 \\
119\end{array}$ & $\begin{array}{l}30.1 \\
43.8\end{array}$ & $\begin{array}{l}1.65 \\
1.54\end{array}$ & $\begin{array}{l}175 \\
183\end{array}$ & $\begin{array}{l}1,190 \\
1,220\end{array}$ & $\begin{array}{l}2,500 \\
2,500\end{array}$ & $\begin{array}{l}141 \\
146\end{array}$ & $\begin{array}{l}3.59 \\
3.47\end{array}$ & $\begin{array}{l}173 \\
159\end{array}$ \\
\hline \multirow[t]{2}{*}{5.} & $\mathrm{C}$ & $\begin{array}{l}112 \\
112\end{array}$ & $\begin{array}{l}19.3 \\
19.3\end{array}$ & $\begin{array}{l}1.21 \\
1.06\end{array}$ & $\begin{array}{l}135 \\
119\end{array}$ & $\begin{array}{l}865 \\
762\end{array}$ & $\begin{array}{l}2,000 \\
2,000\end{array}$ & $\begin{array}{l}119 \\
132\end{array}$ & $\begin{array}{l}2.16 \\
2.10\end{array}$ & $\begin{array}{l}116 \\
102\end{array}$ \\
\hline & I & $\begin{array}{r}109 \\
88\end{array}$ & $\begin{array}{l}17.3 \\
31.7\end{array}$ & $\begin{array}{l}1.43 \\
1.26\end{array}$ & $\begin{array}{l}156 \\
111\end{array}$ & $\begin{array}{r}1,040 \\
770\end{array}$ & $\begin{array}{l}2,000 \\
2,400\end{array}$ & $\begin{array}{l}125 \\
136\end{array}$ & $\begin{array}{l}2.69 \\
2.57\end{array}$ & $\begin{array}{l}143 \\
131\end{array}$ \\
\hline \multirow[t]{2}{*}{6.} & $\mathrm{G}$ & $\begin{array}{l}83 \\
85\end{array}$ & $\begin{array}{l}26.7 \\
30.1\end{array}$ & $\begin{array}{l}1.10 \\
1.07\end{array}$ & $\begin{array}{l}91 \\
91\end{array}$ & $\begin{array}{l}478 \\
511\end{array}$ & $\begin{array}{l}1,880 \\
1,880\end{array}$ & $\begin{array}{l}108 \\
111\end{array}$ & $\begin{array}{l}1.84 \\
1.83\end{array}$ & $\begin{array}{l}89 \\
93\end{array}$ \\
\hline & 1 & $\begin{array}{l}77 \\
86\end{array}$ & $\begin{array}{l}38.7 \\
38.3\end{array}$ & $\begin{array}{l}1.14 \\
1.19\end{array}$ & $\begin{array}{r}88 \\
102\end{array}$ & $\begin{array}{l}507 \\
589\end{array}$ & $\begin{array}{l}2,070 \\
2,260\end{array}$ & $\begin{array}{l}113 \\
117\end{array}$ & $\begin{array}{l}1.99 \\
2.15\end{array}$ & $\begin{array}{l}102 \\
106\end{array}$ \\
\hline \multirow[t]{2}{*}{7.} & $\mathrm{C}$ & $\begin{array}{l}91 \\
96\end{array}$ & $\begin{array}{l}34.2 \\
28.3\end{array}$ & $\begin{array}{l}1.15 \\
1.05\end{array}$ & $\begin{array}{l}105 \\
100\end{array}$ & $\begin{array}{l}460 \\
505\end{array}$ & $\begin{array}{l}2,940 \\
2,940\end{array}$ & $\begin{array}{l}114 \\
122\end{array}$ & $\begin{array}{l}2.15 \\
2.10\end{array}$ & $\begin{array}{l}83 \\
86\end{array}$ \\
\hline & $I$ & $\begin{array}{r}96 \\
102\end{array}$ & $\begin{array}{l}41.7 \\
40.0\end{array}$ & $\begin{array}{l}1.37 \\
1.32\end{array}$ & $\begin{array}{l}132 \\
134\end{array}$ & $\begin{array}{l}640 \\
662\end{array}$ & $\begin{array}{l}3,420 \\
3,300\end{array}$ & $\begin{array}{l}120 \\
119\end{array}$ & $\begin{array}{l}2.70 \\
2.57\end{array}$ & $\begin{array}{l}107 \\
107\end{array}$ \\
\hline \multirow[t]{2}{*}{8.} & $\mathrm{C}$ & $\begin{array}{l}115 \\
107\end{array}$ & $\begin{array}{l}36.5 \\
36.1\end{array}$ & $\begin{array}{l}1.44 \\
1.52\end{array}$ & $\begin{array}{l}144 \\
163\end{array}$ & $\begin{array}{l}702 \\
815\end{array}$ & $\begin{array}{l}2,050 \\
2,050\end{array}$ & $\begin{array}{l}131 \\
131\end{array}$ & $\begin{array}{l}3.17 \\
3.34\end{array}$ & $\begin{array}{l}118 \\
127\end{array}$ \\
\hline & I & $\begin{array}{l}96 \\
93\end{array}$ & $\begin{array}{l}33.2 \\
29.5\end{array}$ & $\begin{array}{l}1.46 \\
1.62\end{array}$ & $\begin{array}{l}146 \\
162\end{array}$ & $\begin{array}{l}793 \\
910\end{array}$ & $\begin{array}{l}2,370 \\
2,370\end{array}$ & $\begin{array}{l}154 \\
154\end{array}$ & $\begin{array}{l}3.77 \\
4.10\end{array}$ & $\begin{array}{l}133 \\
149\end{array}$ \\
\hline \multirow[t]{2}{*}{9.} & C & $\begin{array}{l}126 \\
117\end{array}$ & $\begin{array}{l}27.7 \\
25.6\end{array}$ & $\begin{array}{l}0.74 \\
0.86\end{array}$ & $\begin{array}{r}93 \\
101\end{array}$ & $\begin{array}{l}492 \\
523\end{array}$ & $\begin{array}{l}2,540 \\
2,310\end{array}$ & $\begin{array}{l}137 \\
126\end{array}$ & $\begin{array}{l}2.12 \\
2.26\end{array}$ & $\begin{array}{l}82 \\
94\end{array}$ \\
\hline & $I$ & $\begin{array}{l}94 \\
98\end{array}$ & $\begin{array}{l}27.8 \\
39.8\end{array}$ & $\begin{array}{l}0.99 \\
1.15\end{array}$ & $\begin{array}{r}93 \\
113\end{array}$ & $\begin{array}{l}540 \\
638\end{array}$ & $\begin{array}{l}2,890 \\
3,350\end{array}$ & $\begin{array}{l}146 \\
143\end{array}$ & $\begin{array}{l}3.06 \\
3.46\end{array}$ & $\begin{array}{l}121 \\
137\end{array}$ \\
\hline \multirow[t]{2}{*}{10.} & $\mathrm{C}$ & $\begin{array}{l}93 \\
88\end{array}$ & $\begin{array}{l}30.5 \\
25.8\end{array}$ & $\begin{array}{l}1.07 \\
1.03\end{array}$ & $\begin{array}{l}99 \\
91\end{array}$ & $\begin{array}{l}556 \\
511\end{array}$ & $\begin{array}{l}1,000 \\
1,000\end{array}$ & $\begin{array}{r}96 \\
103\end{array}$ & $\begin{array}{l}2.42 \\
2.50\end{array}$ & $\begin{array}{l}141 \\
136\end{array}$ \\
\hline & I & $\begin{array}{l}101 \\
101\end{array}$ & $\begin{array}{l}32.7 \\
32.7\end{array}$ & $\begin{array}{l}1.24 \\
1.21\end{array}$ & $\begin{array}{l}125 \\
122\end{array}$ & $\begin{array}{l}748 \\
730\end{array}$ & $\begin{array}{l}1,750 \\
1,750\end{array}$ & $\begin{array}{l}139 \\
139\end{array}$ & $\begin{array}{l}4.08 \\
3.96\end{array}$ & $\begin{array}{l}175 \\
171\end{array}$ \\
\hline
\end{tabular}

(Units described in text)

Key: $\mathrm{C}=$ control, $\quad \mathrm{I}=$ immediate 
SWI : $\quad 68-186 \mathrm{~mm} . \mathrm{Hg}-\mathrm{ml} . / \mathrm{Kg}$.

SPI : $\quad 503-1,220 \mathrm{~mm} . \mathrm{Hg}-\mathrm{ml} . / \mathrm{Kg}$. sec.

$\mathrm{dP} / \mathrm{dt}: \quad 1,670-3,420 \mathrm{~mm} . \mathrm{Hg} / \mathrm{sec}$.

HR : $\quad 113-177$ beats $/ \mathrm{min}$.

CO : $\quad$ 1.99-4.10 L./min.

MER : $95-214 \mathrm{ml} . / \mathrm{sec}$.

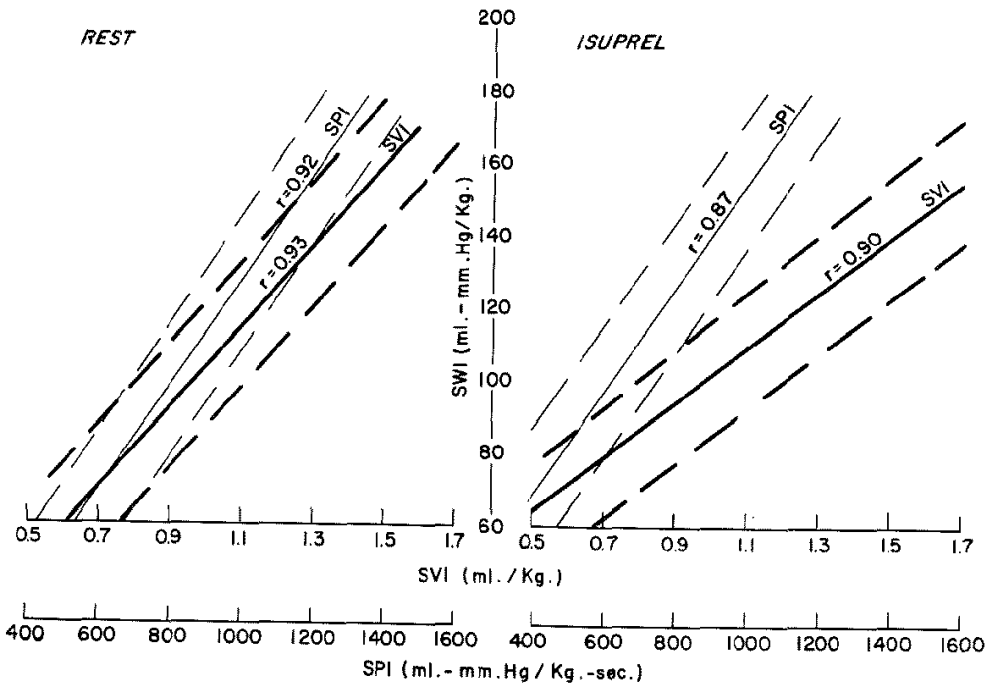

Fig. 1. Comparison of stroke power and stroke volume indices against stroke work index. Note that slope of SPI is unaltered after Isuprel but SVI diminishes.

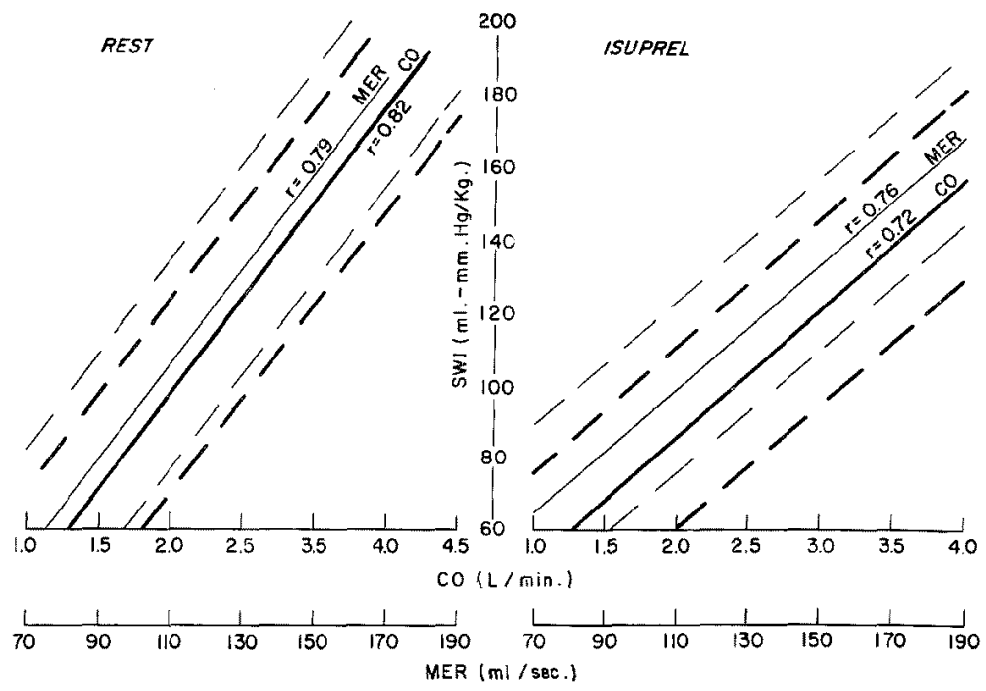

Fig. 2. Comparison of cardiac output (CO) with mean ejection rate (MER) at rest and after Isuprel. Note excellent correlation. 
The regression analyses relating other cardiac performance measurements to SWI are presented in Tables II and III. These measurements are listed in their decreasing order of correlation. The highest correlation obtained in this study was between the SVI and SWI (Fig. 1). Under resting conditions the correlation coefficient was 0.93 and with infusion of isoproterenol 0.90 . The slopes of these regressions, however, were altered with stress. The SPI also

Table II. Resting Data

\begin{tabular}{lll}
\hline SWI $( \pm 17.6)=108.8(\mathrm{SVI})-3.9$ & $\mathrm{r}=0.93$ \\
$\mathrm{SWI}( \pm 18.2)=0.146(\mathrm{SPI})+17.8$ & $\mathrm{r}=0.92$ \\
$\mathrm{SWI}( \pm 26.9)=62.5(\mathrm{CO})-27.1$ & $\mathrm{r}=0.82$ \\
$\mathrm{SWI}( \pm 29.1)=1.12(\mathrm{MER})-8.8$ & $\mathrm{r}=0.79$ \\
$\mathrm{SWI}( \pm 42.3)=0.04(\mathrm{dP} / \mathrm{dt})+47.1$ & $\mathrm{r}=0.44$ \\
$\mathrm{SWI}( \pm 42.3)=1.39(\mathrm{MSP})-22.6$ & $\mathrm{r}=0.44$ \\
$\mathrm{SWI}( \pm 45.4)=-0.70(\mathrm{HR})+212.0$ & $\mathrm{r}=0.26$ \\
$\mathrm{SWI}( \pm 46.0)=1.68(\mathrm{TTI}) \pm 80.25$ & $\mathrm{r}=0.22$
\end{tabular}

Table III. Isoproterenol

\begin{tabular}{lll}
\hline $\mathrm{SWI}( \pm 16.6)=76.4$ & $(\mathrm{SVI})+25.6$ & $\mathrm{r}=0.90$ \\
$\mathrm{SWI}( \pm 18.3)=0.144(\mathrm{SPI})+11.1$ & $\mathrm{r}=0.87$ \\
$\mathrm{SWI}( \pm 26.0)=34.8(\mathrm{CO})+16.2$ & $\mathrm{r}=0.72$ \\
$\mathrm{SWI}( \pm 24.4)=0.850(\mathrm{MER})+6.53$ & $\mathrm{r}=0.76$ \\
$\mathrm{SWI}( \pm 28.8)=0.0156(\mathrm{dP} / \mathrm{dt})+85.3$ & $\mathrm{r}=0.26$ \\
$\mathrm{SWI}( \pm 37.3)=-0.225(\mathrm{MSP})+144$ & $\mathrm{r}=0.08$ \\
$\mathrm{SWI}( \pm 33.6)=-0.777(\mathrm{HR})+233$ & $\mathrm{r}=0.43$ \\
$\mathrm{SWI}( \pm 34.1)=1.87(\mathrm{TTI})+64.4$ & $\mathrm{r}=0.41$
\end{tabular}

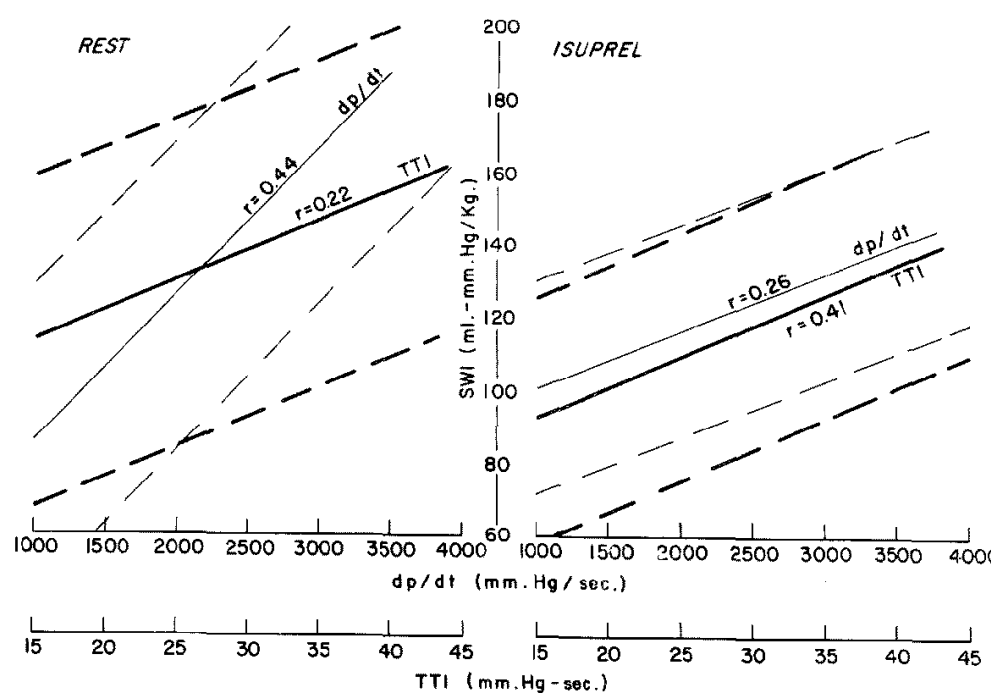

Fig. 3. Comparison of maximum systolic pressure derivative ( $\mathrm{dP} / \mathrm{dt}$ ) and tension time index (TTI) to the stroke work index. Note poor correlation. 


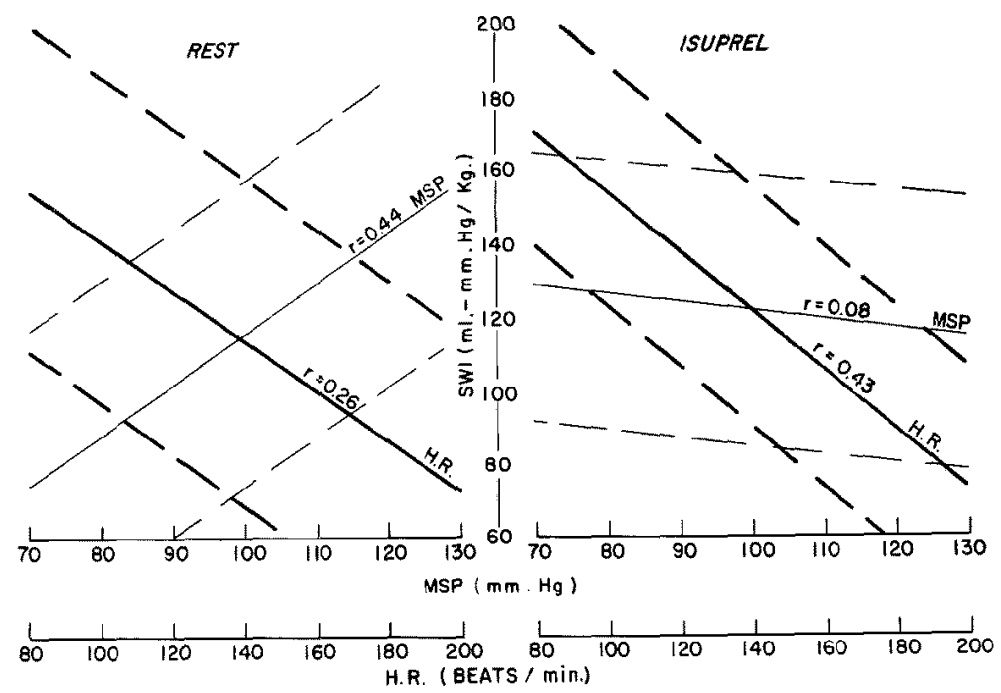

Fig. 4. Comparison of mean systolic pressure (MSP) and heart rate to SWI. Note poor correlation.

showed an extremely high correlation with the SWI (Fig. 1) under resting conditions $(\mathbf{r}=0.92)$. The relationship as well as the slope of the correlation was maintained with infusion of isoproterenol $(r=0.87)$.

Cardiac output under resting conditions showed a good correlation with the stroke work index $(r=0.82)$ and this correlation was preserved after isoproterenol administration $(\mathrm{r}=0.72)$. These correlations are illustrated in Fig. 2.

The systolic pressure derivative was poorly related to SWI under resting conditions $(r=0.44)$, but with administration of isoproterenol no correlation was found $(r=0.08)$. The TTI was also poorly correlated with SWI both at rest $(r=0.22)$ and after isoproterenol $(r=0.41)$. These correlations are shown in Fig. 3. There were no significant relationships between SWI and HR or MSP under resting conditions or after isoproterenol infusion (Fig. 4).

\section{Discussion}

Although the various parameters available for gauging cardiac performance are often used interchangeably, they do not define the same properties of myocardial contraction. Direct comparison of these measurements in either resting or stress states has not bccn quantitated critically. In the parameters studied here it was possible to compare resting values to those obtained after imposition of stress.

Regression analyses of these data indicate that these performance measure- 
ments are not all directly related. Only the flow phenomena, SWI, SVI, SPI, CO and MER were closely correlated. Such correlations were not obtained with the other parameters of the study: $\mathrm{dP} / \mathrm{dt}, \mathrm{MSP}, \mathrm{HR}$ and TTI.

An attempt was made to reclassify the data by grouping rate dependent functions (dP/dt, HR, MER, SPI) and pressure dependent functions (MSP, $\mathrm{dP} / \mathrm{dt}, \mathrm{TTI}$ ). Of the rate dependent functions the only correlations which bordered on significance were $\mathrm{dP} / \mathrm{dt}$ and MER ; and of the pressure dependent functions, $\mathrm{dP} / \mathrm{dt}$ and TTI.

Description of cardiac function curves classically has been accomplished by comparing stroke volume or stroke work to end diastolic ventricular pressure. More recently the time tension index,${ }^{3)}$ mean ejection rate ${ }^{4)}$ and the ventricular pressure derivative ${ }^{51}$ as performance measurements have been utilized. Braunwald ${ }^{6,7)}$ found that function curves could be constructed equally well with each of these parameters. The data presented here, however, indicate that these functions cannot always be used interchangeably and that the experimental conditions modify the interrelationships between cardiac performance measurements.

\section{Summary and Conclusions}

A comparison of the cardiovascular performance measurements in current use was investigated. Ancsthetizcd animals were studied at rest, and after alteration of hemodynamics by administration of isoproterenol. The best correlations were obtained between the flow-related parameters: stroke work, stroke power, stroke volume, cardiac output and mean ejection rate, in that order. The tension time index, ventricular pressure derivative, heart rate and mean systolic pressure showed no consistent correlations either with the stroke index or between each other. It is concluded that the effects of altered hemodynamics must be considered before using different cardiac performance measurements interchangeably.

\section{REFERENCES}

1. Sarnoff, S. J. and Mitchell, J.H.: Am. J. Med. 30:747, 1961.

2. Rushmer, R. F., Van Citters, R. L., and Franklin, D. L.: Circulation 27 : 118, 1963.

3. Sarnoff, S.J., Braunwald, G. H., Welch, R. B., Jr., Case, W., Stainsby, N., and Marcuz, R.: Am. J. Physiol. 192: 1958.

4. Braunwald, E., Sarnoff, S.J., and Stainsby, W. N.: Circulat. Res. 6: 319, 1958.

5. Reeves, T. and Hefner, J.: Am. Heart J. 60: 746, 1960.

6. Braunwald, E., Frahm, C. J., and Ross, J., Jr.: J. Clin. Invest. 40 : 1882, 1961.

7. Braunwald, E.: Brit. Heart J. $27: 1,1965$.

8. Rushmer, R. F., Smith, O., and Franklin, D. : Circulat. Res. 7: 602, 1959.

9. Snedecor, G. W.: Statistical Methods Applied to Experiments in Agriculture and Biology, ed. 5, Ames Iowa, Iowa State College Press, 1956. 